Annales UMCS

Informatica

Lublin-Polonia

Sectio AI

http://www.annales.umcs.lublin.pl/

\title{
The Concept and Security Analysis of Wireless Sensor Network for Gas Lift in Oilwells
}

\author{
Bartlomiej Bielecki $^{1}$, Bogdan Ksiezopolski ${ }^{1,2}$, Andrzej Krajka ${ }^{1}$, Adam \\ Wierzbicki ${ }^{1,2}$ \\ ${ }^{1}$ Institute of Computer Science, Maria Curie-Sklodowska University, \\ pl. M. Curie-Sklodowskiej 5, 20-031 Lublin, Poland \\ ${ }^{2}$ Polish-Japanese Institute of Information Technology, \\ Koszykowa 86, 02-008 Warsaw, Poland
}

\begin{abstract}
Pipelines, wellbores and ground installations are permanently controlled by sensors spread across the crucial points in the whole area. One of the most popular techniques to support proper oil drive in a wellbore is a Gas Lift. In this paper we present the concept of using wireless sensor network (WSN) in the oil and gas industry installations. Assuming that Gas Lift Valves (GLVs) in a wellbore annulus are sensor controlled, the proper amount of injected gas should be provided. In a ground installation, the optimized amount of loaded gas is a key factor in the efficient oil production. This paper considers the basic foundations and security requirements of WSN dedicated to Gas Lift Installations. Possible attack scenarios and their influence on the production results are shown as well.
\end{abstract}

\section{Introduction}

Oil and Gas production is based on fundamental physical properties. The pressure difference between the ground installation - wellhead and the well bottom pushes the fluid uphill, towards the surface. The density of oil has the influence on the production results. One of the most popular techniques to reduce oil density in a wellbore is a Gas Lift. In the USA about 10 percent of wells use the intermittent or permanent installation providing natural gas through valves into a wellbore. Gas Lift systems are divided into two sections: underground and surface. In the underground area, Gas Lift Valves (GLVs) provide the natural gas into the wellbore. They are set in producers labs, to open under the dedicated pressure differences. Inside a GLV orifices provide 
gas into a tubing, causing higher pressure difference between a wellhead and a bottom hole. To change the properties, valves are retrieved and this is a time consuming and expensive method. On the surface, especially for the multiwell installation, the gas distribution should take the optimization problems under the consideration. Proper amount of injected gas has the influence on the production results[13].

In the Oilwell industry the temperature and pressure gauges are commonly used. Unfortunately, these devices could not be placed across the whole wellbore, so Distributed Temperature Sensing (DTS) systems are used [9]. For the survey, thin fiber optic cables made of silicon dioxide with the amorphous solid structure are used. Physical properties as temperature or pressure can affect characteristics of light transmission in the cable. In a fiber, while a photon affects the crystal it is annihilated. The Rayleigh scattering is described as the photon annihilation whiles having a contact with the crystal and creation of another photon [15]. The energy balance between the scattered protons is not equal and it can be observed in the spectrum with the Stokes and anti-Stokes lines. The DTS systems have a few drawbacks. The fiber optic cable can be damaged, and the replacement takes an unnecessary time to fix the problem by replacing or splicing the cable. The temperature data usually cause small disturbation in the places where cable is clamped to the wellbore. It makes the automatic analysis tough to perform and can be interpreted as genuine effects or unexpected leaks. DTS demands the equipment for the data collection and a big spool of fiber optic cable. All these things should be shipped to provide the proper data acquisition.

The main contribution of this paper is to propose the concept of monitoring system based on Wireless Sensor Network. The presented idea is the novel one and is not discussed in literature. The traditional Gas Lift properties are set in laboratories prior to the well completion. Once the calculation is wrong, it is nearly impossible to improve the system without decompletion [13]. Wireless sensor network can be responsible for GLVs communication, to manage the proper lift. Every wellbore at an oilfield may be connected to the gas wireless distributing system. These facts determine the idea of dynamic WSN dedicated to the gas lift managing and monitoring. Another contribution of this paper is to show the security requirements and potential impact of the attacks for WSN for Gas Lift in Oilwells. The security attacks on WSN can damage the system by changing the temperature and pressure data. As a result, the production can be totally corrupted or disturbed. Once the attack reaches a safety valve, the results are highly dangerous, even devastating. At the surface part of the completion, any attack deregulates the gas diversion. Regarding the study about potential impact in the case of attacks on the WSN monitoring system, the authors extended the simulation model to the single phase annulus calculation [3]. 


\section{Wireless Sensor Network in a Wellbore}

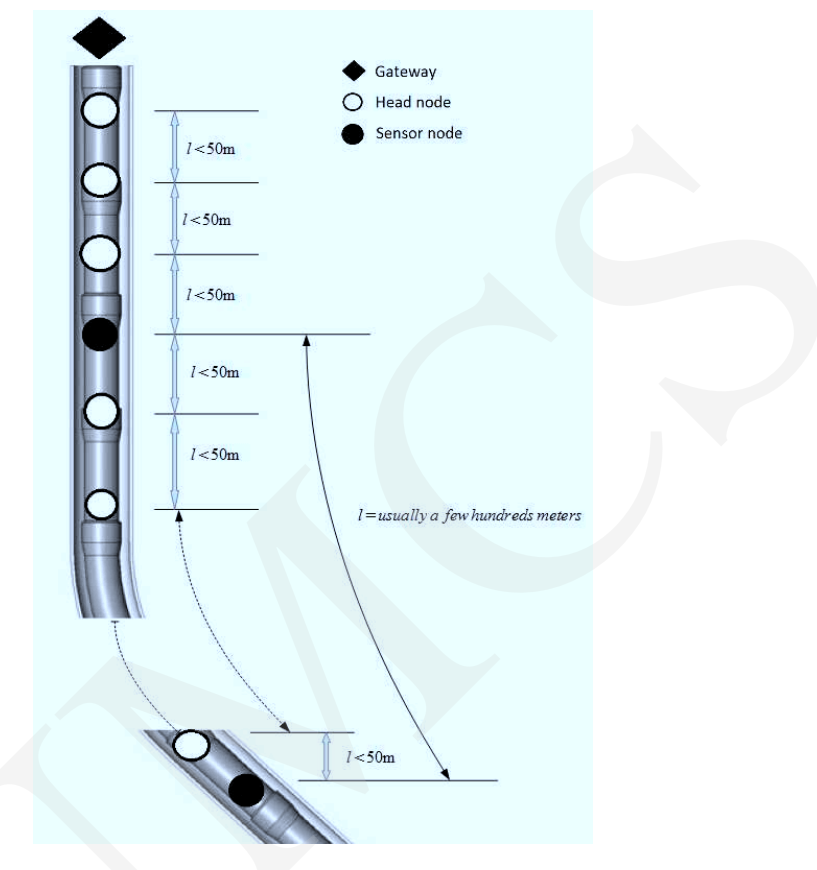

FIG. 1. WSN topology in a wellbore

In the literature, different network topologies for Wireless Sensor Network can be found $[\mathbf{1}, \mathbf{5}, \mathbf{1 1}]$. The network topologies can include various types of nodes which have a different function. The most common are:

- sensing node - it samples a kind of data (i.e. temperature, acceleration, etc.);

- head node - it collects data from sensing nodes and forwards it to the gateway;

- gateway (base station) - this node is directly connected to a server and forwards the received data to it (i.e. via the serial port).

One can enumerate the main types of topology as follows:

- star topology, consisting of the gateway node and sensing nodes only;

- tree topology, consisting of all three types of nodes where head nodes stand between the sensing nodes and the gateway;

- mesh topology, similar to tree topology, but the head nodes can be connected to other head nodes directly.

In this paper we consider a sensor network, which is built of three kinds of sensors: sensing nodes (GLVs), head nodes and the gateway. The network topology is shown in 
Fig. 1. The distance between each sensor is estimated as $50 \mathrm{~m}$, and the well depth usually reaches a few kilometres, this is tree topology with only one gateway. The working sensors transmit the data to the gateway sensor which is situated at the wellhead. In the Gas Lift point, the pressure sensor is responsible for data survey. Other sensors are mandatory in the terms of communication between GLVs and gateways on the surface. The linear structure of sensors deployment in the wellbore keeps the monitoring more accurate. In the completion lots of additional and safety devices is fitted. By putting sensors in the proper places, not only gas lift can be controlled, also the safety issues can be reduced. To estimate the exact sensor distribution, the further studies are needed. Currently, the assumption that every 50 meters the sensor is placed has been made, according to the measured depth. The meshing is not necessary, it should be constant and it is completion dependable.

WSN in a wellbore may be a part of monitoring only, or a decisive system which takes the responsibilities for the production process. Regarding the system automation three levels of autonomy are considered: full, semi full, none. Full automation is when GLVs are gas lifted under the set pressure circumstances. The temperature in the wellbore is checked by sensors and the results are sent to the system, which is responsible for the pressure calculation. If the pressure conditions are adequate, the algorithm makes a decision which valves should stay open. Once the gas is lifted, the algorithm checks the conditions and reduce the injection to avoid the turbulent flow. The amount of gas in this wellbore is the variable which is the function of the whole amount of gas in the system. Safety procedures are controlled by the sensor, though they are wireline connected, which supports the additional control barrier. Semi-full automation gives the exception for safety procedures and the gas injection is controlled by WSN. The unautomated system is being developed to check the physics conditions by monitoring and control. It creates the option to analyze the production process and the comparison with the classic form of well managing.

\section{Security Requirements Analysis}

Realization of electronic processes requires fulfilment of many technological standards so while projecting the systems one can take care of different security services. The large list of them is presented and discussed in the articles $[\mathbf{5}, \mathbf{1 2}]$. Realization of WSN for Oilwells requires fulfilment of several security services. In this section we would like to enumerate the most important ones and provide justification for our choice.

- Confidentiality of data. The oilfield data has the priority regarding the data confidentiality. A few reasons exist in the background. First of all, no one is interested to uncover the crucial production data. In some cases the oil production has been kept on the proper level which not necessarly means the highest. It is strongly demanding as fas as financial and other external reasons are concerned. 
- Integrity of data. One of the most important thread, is to capture the sampled data in the network and modify them. It can damage the production or destroy safety procedures.

- Authorization of nodes. Another attack can be focused on impersonation of the sensing nodes. In that situation the sensing data can be modified, too. In this situation, the production can also be damaged.

- Availability of nodes. This service is especially important for sensing nodes because in the GLV there can be located only one node. The head nodes are not so crucial as the sensing nodes because they can be replicated.

\section{Modelling the Impact of the Data Modification Attack for WSN for Gas Lift in Oilwells}

Assuming that the malicious sensor changes the data in the system a few cases have been analyzed here. The important thread happens once the attack distorts pressure measurements in the annulus. As a result, the GLV changes the lifting sequence causing undesirable situations as slug flow, production problems even temporary block of the fluid drive. The presented results were simulated using the model which was meant to estimate the production results and thermodynamic conditions in a wellbore.

There are many factors determining the production calculations such as: well trajectory, reservoir data, casing and tubing data, flow types, heat transfer, bubble point pressure, viscosity, velocity, density, volume factor, compressibility, heat capacity, temperature, pressure. The drive in the tubing is usually calculated from different approaches for Darcy's Law and its extensions. The Darcy equation presented by Peaceman [10] is as follows:

$$
\frac{\delta}{\delta t}\left(\phi \sum_{j=1}^{n_{p}} \xi_{j} S_{j} x_{i j}\right)-\nabla \bullet\left[\sum_{j=1}^{n_{p}} \xi_{j} x_{i j} \lambda_{j}\left(\nabla P_{j}-\varrho_{j} D\right)\right]-\frac{q_{i}}{V_{b}}=0
$$

where: $g_{i}$ - molar flow, $\phi$ - molar density of $j$-phase, $\xi$ - phase saturation, $V_{b}$ - phase molar volume, $x_{i j}$ - flux vector, $\mathrm{P}$ - pressure, $n_{p}$ phase number, $\varrho$ - density, $\lambda$ - mobility ratio.

To the simulation model based on equation 1, the authors added the single phase gas correlations dedicated to the annulus. Based on Fanning correlation [3]:

$$
\Delta P_{f}=\frac{2 f \rho V^{2} L}{g_{c} D}
$$

where: f - Fanning friction factor (function of Reynolds number), $\rho$ - density, v average velocity, L - length of pipe section, $g_{c}$ - gravitational constant, D - inside diameter of pipe. 
The Fanning friction factor is calculated using the formula[4]:

$$
\frac{1}{\sqrt{f}}=-4.0 \log \left[0.2698\left(\frac{k}{D}\right)-\frac{5.0452}{R e} \log \left\{0.3539\left(\frac{k}{D}\right)^{1.1098}+\frac{5.8506}{R e^{0.8961}}\right\}\right]
$$

where: $\mathrm{f}$ - friction factor, $\mathrm{k}$ - absolute roughness, $\frac{k}{D}$ - relative roughness, Re - Reynolds number.

The single-phase friction factor clearly depends on the Reynolds number, which is a function of the fluid density, viscosity, velocity and pipe diameter. The friction factor is valid for single-phase gas or liquid flow, as their very different properties are taken into account in the definition of Reynolds number[2]:

$$
R e=\frac{\varrho V D}{\mu}
$$

where: $\rho$ - density, $\mu$ - viscosity.

This correlation has been chosen to study pressure difference between tubing and the annulus, to estimate the condition for the gas lift.

\subsection{Scenarios}

In the article we are consider three scenarios which simulate the impact of the attack when the working sensors will be impersonated. During the attacks the measurement values are modified which are different and depend on the gas lift. These data are crucial for the system which control the production. That analysis shows the impact level of the attack and can be useful during constructing of the WSN architecture for oilwells. We assume that six sensing nodes are installed located at six depths: $1000 \mathrm{ft}, 2000 \mathrm{ft}, 3000 \mathrm{ft}, 4000 \mathrm{ft}, 4500 \mathrm{ft}, 5000 \mathrm{ft}$. The pressure calculation in the annulus is simplified due to a single phase.

\subsubsection{Scenario 1}

The attack changes the pressure values in the range of gas lifting between 2 and 3 MMScf/D (Fig. 2). The system identifies the gas lift which drops to 1 MMScf/D. The deeper the attack takes place the bigger production drop is observed.

\subsubsection{Scenario 2}

Another simulation shows the attack for the lift between 3 and 4 MMScf/D (Fig.3). Regarding the productivity this is the most desirable amount of gas in the annulus. The system identifies that gas is not distributed to this wellbore. Without the malicious changes, more than 3000 BPD is expected. Meanwhile, the highest drop is observed if the sensor data is overwritten at $4000 \mathrm{ft}$. 
Pobrane z czasopisma Annales AI- Informatica http://ai.annales.umcs.pl

Data: 26/04/2023 06:06:35

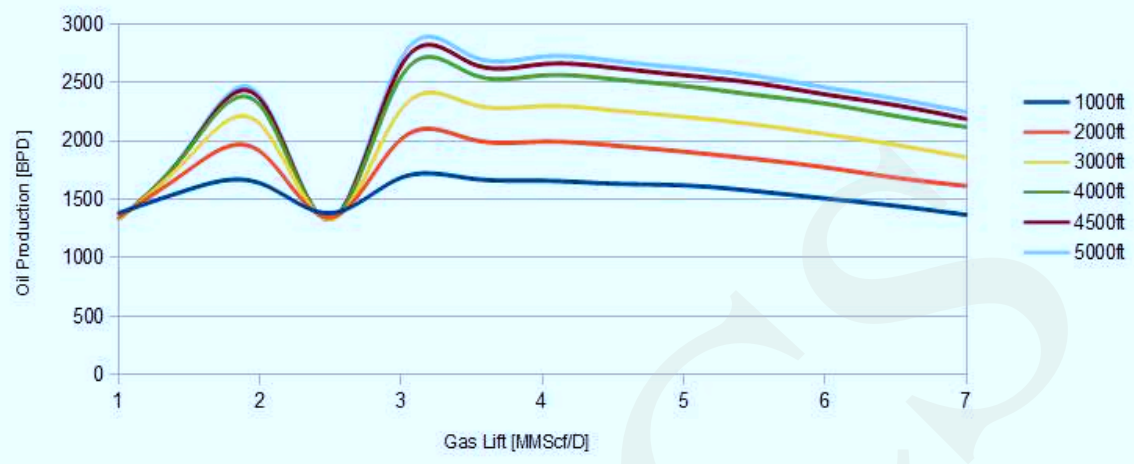

FIG. 2. The attack scenario 1

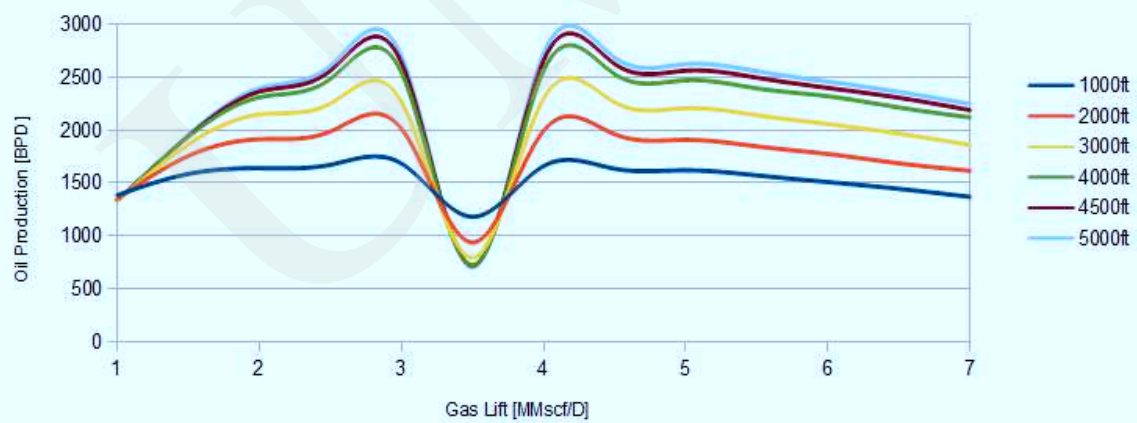

FIG. 3. The attack scenario 2

\subsubsection{Scenario 3}

The thread combined with high amount of gas involved leads to the interesting results. Once the malicious sensor changes the results by adding too much gas to the system, the production stops definitely. In this case the attack took place for in range from 4.5 to 5.5 MMScf/D (Fig.4). Lifting more gas to the system when it is expected, causes significant reduction of oil density in the wellbore. The weight of the column above the GLV pushes the drive towards the bottom, almost stopping the production. 


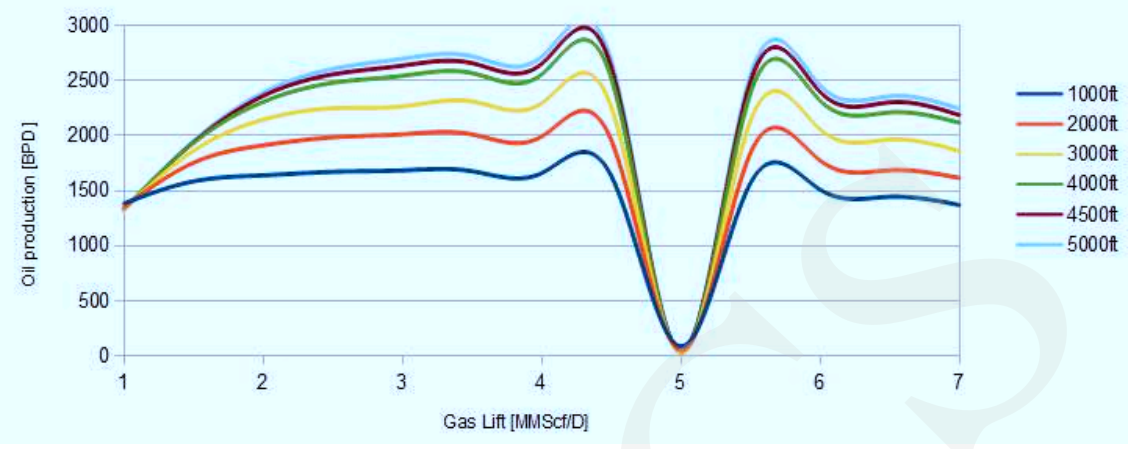

FIG. 4. The attack scenario 3

\section{Conclusions}

In this paper we show the concept of Wireless Sensor Networks architecture which can be applied in oilfields, especially for the Gas Lift monitoring and management. The importance of the security of the oil production is undoubtable and this paper represents the very first approach to this problem. We present the main security services which must be guaranteed for presented idea. In the paper the simulation model showing the effects of the potential attack inside the network which can destabilize the production process was presented. It has been showed that the more gas during the lift is in the annulus, the higher risk of stopping the production takes place. Furthermore, the deeper the GLV operates, greater effect the attack has. The information about the threat level and potential attacks impact can be used for introducing the adaptable security $[6,7]$ and Quality of Protection analysis.

In the future work we would like to analyse the security mechanisms which should be fulfilled to satisfy the enumerated security requirements. Next, the performance analysis will be prepared in the terms of the used security mechanisms for the lifetime of the WSN. To achieve this the QoP model will be prepared in Quality of Protection Modelling Language (QoP-ML) [8] and simulated in the AQoPA tool [14].

\section{References}

[1] Baronti P., Pillai P., Chook V.W., Chessa S., Gotta A., Fu Y.F., Wireless sensor networks: a survey on the state of the art and the 802.15.4 and ZigBee standards, Computer Communication, 30 (2007): 1655-1695.

[2] Chen, N. H., An Explicit Equation for Friction Factor in Pipe, Ind. Eng. Chem. Fund. (1979).

[3] Chen N. H., Ind. Eng. Chem. Fundamen., 18 (3) (1979): 296-297.

[4] Concha, F., Settling velocities of particulate systems 15: Velocities in turbulent Newtonian flows. International Journal of Mineral Processing, 88 (3-4) (2008): 89-93. 
[5] Ksiezopolski B., Kotulski Z., On scalable security model for sensor networks protocols, 22nd CIBW78 Conference Information Technology in Construction, Dresden (2005): 463-469.

[6] Ksiezopolski B., Z.Kotulski Z., Szalachowski P., Adaptive approach to network security - Springer: CCIS, Computer Networks, 39 (2009): 233-241.

[7] Ksiezopolski B., Kotulski Z., Szalachowski P., On QoP method for ensuring availability of the goal of cryptographic protocols in the real-time systems, Conference: European Teletraffic Seminar (2011).

[8] Ksiezopolski B., QoP-ML: Quality of protection modeling language for cryptographic protocols. Computers \& Security, 31(4) (2012): 569-596.

[9] Liaqat A., Russell E. B., Gutierrez A. A., Yosmar G., Using distributed temperature sensing (DTS) technology in acid gas injection design, Acta Geotechnica, 9(1) (2014): 135-144.

[10] Peaceman D.W., Interpretation of well-block pressures in numerical reservoir simulation with nonsquare grid blocks and anisotropic permeability. SPE J. (1983): 531-543.

[11] Rusinek D., Ksiezopolski B., On effect of security and communication factors on the reliability in Wireless Sensor Networks Journal of Sensor and Actuator Networks, 3(1) (2014): 81-94.

[12] Szalachowski P., Ksiezopolski B., Kotulski Z., On authentication method impact upon data sampling delay in Wireless Sensor Networks Communications in Computer and Information Science, Computer Networks (2010): 280-289.

[13] Takacs G., Gas Lift Manual, PennWell Books (2005).

[14] The official web page of the QoP-ML project: http://www.qopml.org.

[15] Zou J., Wang S., Yang Z., Distributed Temperature Measurement Based on Single-Channel AntiStokes Light, International Journal of Infrared and Millimeter Waves, 21(5) (2000): 793-803. 